\title{
TITLE
}

\section{Influence of Temporal Parameters of DCE-MRI on the Quantification of Heterogeneity in Tumor Vascularization.}

\author{
$\underline{\text { Crombé }}^{1,2}$, Saut $\mathrm{O}^{2}$, Guigui ${ }^{1}$, Italiano $\mathrm{A}^{3}$, Buy $\mathrm{X}^{1}$, $\underline{\text { Kind }}^{1}$.
}

1. Department of Radiology, Institut Bergonié, Comprehensive Cancer Center, Bordeaux, France.

2. University of Bordeaux, IMB, UMR CNRS 5251, INRIA Project Team Monc, Talence, France.

3. Department of Medical Oncology, Institut Bergonié, Comprehensive Cancer Center, Bordeaux, France.

\begin{abstract}
:
Background: Evaluating heterogeneity in tumor vascularization through texture analysis could improve predictions of patients' outcome and response evaluation.

Purpose: To investigate the influence of temporal parameters on texture features extracted from DCE-MRI parametric maps.
\end{abstract}

Study type: Prospective cross-sectional study

Subjects: 25 adults with soft-tissue sarcoma (STS), median age: 68 years.

Field Strength/Sequence: DCE-MRI acquisition using a CAIPIRINHA-DixonTWIST-VIBE sequence at $1.5 \mathrm{~T}$ (temporal resolution: $2 \mathrm{~s}$, duration: $5 \mathrm{~min}$ ).

Assessment: The area under time-intensity curve (AUC) and $\mathrm{K}^{\operatorname{tans}}$ maps were generated for several temporal resolution $(\mathrm{dt}=2 \mathrm{~s}, 4 \mathrm{~s}, 6 \mathrm{~s}, 8 \mathrm{~s}, 10 \mathrm{~s}, 12 \mathrm{~s}, 20 \mathrm{~s})$ and scan durations ( $\mathrm{T}=3 \mathrm{~min}, 4 \mathrm{~min}, 5 \mathrm{~min}$ for a $6 \mathrm{~s}$-sampling) by downsampling and truncating the initial DCE-MRI sequence. Tumor volume was manually segmented and propagated on all parametric maps. Thirty-two first- and second order-texture features were extracted per map to quantify the intra-tumoral heterogeneity.

Statistical Tests: The influence of temporal parameters on texture features was studied with repeated-measures ANOVA (or non-parametric equivalent). The dispersion of each texture feature depending on temporal parameters was estimated with coefficients of variation $(\mathrm{CV})$. The performances of multivariate models to predict the response to chemotherapy (i.e. binary logistic regression based on the baseline texture features) were compared. 
Results: The temporal resolution had a significant influence on 12/32 (37.5\%) and 14/32 (43.8\%) texture features evaluated on AUC and Kras maps, respectively (range of p-values: <0.0001-0.0395). Scan duration had a significant influence on 23/32 $(71.9 \%)$ texture features from $\mathrm{K}^{\text {tans }}$ map (range of p-values: <0.0001-0.0321). Dispersion was high (: mean $\mathrm{CV}>0.5)$ with sampling for $2 / 32(6.3 \%)$ and $10 / 32$ (31.3\%) features from AUC and $\mathrm{K}^{\text {tans }}$ maps, respectively; and with truncating for $6 / 32$ (18.8\%) features from $\mathrm{K}^{\text {tans }}$ map. The AUROC of predictive models ranged from 0.77 $(\mathrm{CI} 95 \%=[0.54-1.00]$, with $\mathrm{dt}=6 \mathrm{~s}-\mathrm{T}=4 \mathrm{~min})$ to $0.90(\mathrm{CI} 95 \%=[0.74-1.00]$, with $\mathrm{dt}=6 \mathrm{~s}-$ $\mathrm{T}=5 \mathrm{~min})$.

Data Conclusion: The values of texture features extracted from DCE-MRI parametric maps can be influenced by temporal parameters, which can lead to variations in performances of predictive models.

\section{Level of evidence: 2}

\section{Technical efficacy: 2}

\section{KEYWORDS}

Radiomics; Texture analysis; oncology; DCE-MRI; Sarcoma; Sequence optimization

\section{ABBREVIATIONS}

AIF: Arterial input function

AUC: Area under curve

AUROC: Area under the receiver operating characteristics curve

CI95\%: 95\% confidence interval

$\mathrm{CV}$ : Coefficient of variation

DCE-MRI: Dynamic contrast-enhanced MRI

FNCLCC: French Federation Nationale des Centres de Lutte Contre le Cancer

FOS: First order statistics

GL: Grey level

GLCM: Grey level occurrence matrix

GLRLM: Grey level run-length matrix

GLSZM: Grey level size zone matrix

$\mathrm{K}^{\text {trans: Influx volume transfer constant }}$

QIBA®: Quantitative Imaging Biomarkers Alliance 
rm: Repeated measures

SD: standard deviation

STS: soft-tissue sarcomas

TSE: turbo spin echo

VIBE: Volume interpolated breath-hold examination

VOI: Volume of interest 


\section{INTRODUCTION}

The extensive quantification of tumor heterogeneity on medical imaging is a growing field of research in oncology referred to as radiomics. The underlying hypothesis of radiomics is that the imaging phenotype of a tumor could reflect its intrinsic molecular identities and aggressiveness $(1,2)$. Texture analyses consist in the mathematical processing of images in order to extract numeric indices that objectively measure the heterogeneity, named texture features. The most commonly encountered ones in the medical literature are $1^{\text {st }}$ order features, which are based on frequency histograms without spatial information, and $2^{\text {nd }}$ order features, which quantify the $2 \mathrm{D}$ and/or 3D rearrangements of voxels of different gray-levels. Predictive radiomics approaches based on texture features, machine learning algorithms and the potential combination with clinical characteristics and other -omics data (i.e. genomics, transcriptomics, proteomics and metabolomics) could help better stratify the therapeutic strategy for cancer patients and evaluate treatment responses (2).

Radiomics approaches can be applied to every imaging modality including dynamic contrast-enhanced MRI (DCE-MRI). DCE-MRI aims at providing a non-invasive macroscopic assessment of tumor perfusion and neo-angiogenesis - a key prooncogenetic process - through the rapid acquisition of a time series of T1-weighted imaging (3). DCE-MRI parameters have been used to discriminate benign from malignant tumors or to monitor treatment efficacy especially anti-angiogenic regimens - the area under the time intensity curve (AUC) and the influx volume transfer constant ( $\mathrm{K}^{\text {trans}}$ ) being the most studied) (4). However, these studies were based on average values of the DCE-MRI parameters that do not reflect the complexity of tumors. Indeed, homogeneous poorly vascularized tumors could have the same mean AUC and $\mathrm{K}^{\text {trans }}$ values as heterogeneous tumors with both hypervascularized and large avascular necrotic areas. Consequently, quantifying the spatial heterogeneity in vascularization of the whole tumor volume from DCE-MRI data may be more informative and realistic. In that sense, radiomics approaches on DCE-MRI have recently shown encouraging results, alone or with other MRI sequences, in order to improve the detection of prostate cancer, to distinguish benign and malignant adnexal masses, to identify relevant molecular subtypes of breast cancers, to detect lymph node metastases in breast cancers, or to predict response to neoadjuvant treatment for rectum, breast and nasopharyngeal cancers (5-12) . Softtissue sarcomas (STS) are malignant mesenchymal tumors with important inter and 
intra-tumoral heterogeneity known to be associated with high grade (13). Previous studies have shown that DCE-MRI could be useful to predict response to chemotherapy in high-grade STS (14-17), as well as radiomics approaches $(16,18$, 19). Thus, it can be hypothesized that combining radiomics and DCE-MRI could enhance the early prediction of tumor response to treatment.

Robust radiomics models require the inclusion of a large number of patients, divided into training, validation and external test cohorts, before being implemented into clinical practice. Pooling data from different centers is necessary but it runs the risk of introducing bias to the values of texture features. Indeed, each step of the radiomics process can introduce variability independently from the intrinsic heterogeneity of the tumor, for instance: MRI field strength, manufacturers, coils, acquisition parameters, segmentation, voxel-size resampling, normalization techniques or grey-level discretization (20-23). Previous studies have demonstrated that temporal parameters (i.e. scan duration and temporal resolution) could significantly modify the ability to discriminate benign from malignant prostate or breast lesions (24-26), but they were based on average values of DCE-MRI indices or morphology of the time-intensity curves. Only one study has focused on the stability of texture features extracted from computed tomography perfusion maps identifying an influence of temporal resolution (27). Hence, data regarding the influence of temporal parameters on texture features extracted from DCE-MRI parametric maps are lacking.

Thus, our aims were to investigate the influence of temporal parameters: (i) on the values of widely used texture features extracted from DCE-MRI parametric maps of STS, (ii) on the dispersion of these parameters, and (iii) on the performance of predictive models for chemotherapy responses. 


\section{MATERIALS AND METHODS}

\section{Patient population}

In this prospective single-center study, the institutional review board waived the requirements of informed patient consent. From November 2017 to June 2018, 30 consecutive adult patients were included as they presented at our sarcoma reference center for the management of a histologically-proven high-grade (according to the French Federation Nationale des Centres de Lutte contre le Cancer [FNCLCC] grading system) STS of the trunk wall or extremities of more than $4 \mathrm{~cm}$, and required a contrast-enhanced MRI for diagnostic and/or therapeutic management. Age, gender, histological type, tumor depth relative to superficial fascia, tumor location, longest diameter, specific treatments and short-term patients' outcome were retrieved from medical records. We defined a good responder as: (i) $<10 \%$ of stainable viable tumor cells on surgical specimen after neo-adjuvant chemotherapy for locally-advanced nonmetastatic STS (28), and (ii) a partial or complete response 6 months after performing DCE-MRI according to RECIST 1.1 criteria for metastatic or inoperable patients, without changing treatment - this last empirical definition being proposed regarding the usual median progression-free survivals of metastatic STS patients (29).

\section{Data acquisition}

Patients underwent MRI scans in the same 1.5T MR-system (MAGNETOM Aera; Siemens Healthineers, Erlangen, Germany) with adapted coils depending on tumor locations and sizes, i.e. 15 channels transmit/receiver coil for knee and extremities and 18 channels transmit/receiver body coil for trunk wall and thighs. Patients were examined in supine position. The protocol followed the Quantitative Imaging Biomarkers Alliance recommendations (QIBA $\left.{ }^{\circledR}\right)$ and consisted in a T1-mapping followed by the DCE-MRI acquisition with the same field-of-view $(350 \times 320 \mathrm{~mm})$ and spatial resolution $\left(1.1 \times 1.1 \times 4 \mathrm{~mm}^{3}\right)$, in order to optimize the conversion of the signal in Gadolinium chelates concentration. We used a CAIPIRINHA (Controlled Aliasing in Parallel Imaging Results in Higher Acceleration) Dixon TWIST (Timeresolved angiography With Stochastic Trajectories) VIBE (Volume Interpolated Breath hold Examination) sequence (30). The T1-mapping used a similar spoiled gradient-echo sequence with variable flip angles $\left(2^{\circ}\right.$ and $\left.15^{\circ}\right)$ and with echo and repetition times of $1.41 \mathrm{~ms}$ and $3.79 \mathrm{~ms}$, respectively. In brief, the principle of TWIST 
is to divide the k-space in a central region, which encodes information about contrast, and a peripheral region, which encodes information about shapes, edges and details and is undersampled (30). The suppression of the fat signal was performed using a Dixon-based water-fat separation (echo times were $2.39 \mathrm{~ms}$ for in-phase and $4.77 \mathrm{~ms}$ for opposite-phase conditions). A repetition time of $6.89 \mathrm{~ms}$ and a flip angle of $25^{\circ}$ were used. The sampling was accelerated with a parallel acquisition technique with an undersampling factor of 2 . The value of the TWIST view-sharing parameters A (i.e. the percentage sizes of the central portion of the k-space) and B (i.e. the percentage sizes of the peripheral portion of the k-space) were $15 \%$ and $20 \%$, respectively. The temporal resolution resulted in $\mathrm{dt}=2 \mathrm{~s}$, except for the first TWIST phase $(6.9 \mathrm{~s}$ for the full k-space sampling). Five phases were acquired before the intra-venous powerinjection of $0.1 \mathrm{mmol} / \mathrm{kg}$ of gadoteric acid (Dotarem, Guerbet, Villepinte, France) at a rate of $2 \mathrm{~mL} / \mathrm{s}$ followed by a $20 \mathrm{~mL}$ flush of $0.9 \%$ of $\mathrm{NaCl}$ solution thanks to a MRIcompatible automatic injector (Sonic Shot 7, Nemoto Kyorindo, Tokyo, Japan). The total scan duration was $5 \mathrm{~min}$ and included 144 phases (full-dataset).

\section{Data reconstruction}

Data reconstruction and post-processing were achieved with Olea Sphere ${ }^{\circledR}$, v3.0 SP14 Software (Olea Medical, La Ciotat, France) by a senior radiologist with 7 years of experience in MRI blinded to clinical data. First, motion artifacts were systematically corrected with a rigid body co-registration method. For each patient, the full-dataset was downsampled and truncated to obtain the datasets to assess the effect of scan duration and temporal resolution. In any case, the 5 first phases were kept in the datasets to ensure a similar baseline for all maps. In total, 7 DCE-MRI datasets per patient were generated to evaluate temporal resolution, namely: $\mathrm{dt}=2 \mathrm{~s}$ (raw data), 4s, 6s, 8s, 10s, 12s, 20s, with a scan duration of $5 \mathrm{~min}$. Three DCE-MRI datasets were generated to evaluate the influence of scan duration, namely: $\mathrm{T}=3 \mathrm{~min}$, $4 \mathrm{~min}, 5 \mathrm{~min}$, all with a temporal resolution of $\mathrm{dt}=6 \mathrm{~s}$. Figure 1 shows how the datasets were built.

The next step consisted in the calculation of the parametric maps using the permeability plug-in of Olea Sphere ${ }^{\circledR}$. First, the senior radiologist manually chose the largest feeding artery within the field-of-view of the raw DCE-MRI dataset, with the exclusion of the first and the last slices to avoid artifacts. A fixed voxel was manually placed in this artery and the arterial input function (AIF) was measured as the average 
of 4 directly adjacent voxels within this artery that demonstrated an arterial enhancement and the less noise. The same artery and the same voxels were used for the other reconstructed datasets. Hence, even if the baseline of the AIF was preserved, the AIF was also downsampled and truncated (Fig. 1). Second, the AIF signal intensity time-course was converted into blood R1 time-course, with the hematocrit = 0.45 and the gadoteric acid relaxivity at $1.5 \mathrm{~T}=3.6 \mathrm{~L} / \mathrm{mmol}^{-1} \mathrm{~s}^{-1}$. We chose to focus on the most widely used parameters: the area under the time-intensity curve (AUC), at 90s (units: $\mathrm{mmol} / \mathrm{L} / \mathrm{s}$ ) and the pharmacokinetic parameter $\mathrm{K}^{\text {trans }}$ (influx volume transfer constant from plasma to extra-vascular, extracellular space, which represents the capillary permeability, units: $\min ^{-1}$ ). $\mathrm{K}^{\text {trans }}$ maps were calculated using the Extended Tofts model (31). Of note, AUC was not evaluated for scan duration because we focused on the first 90 seconds of the time-intensity curve.

Five out of the 30 MRI examinations were excluded due to Dixon fat-water swap artifacts $(n=3)$ and motion artifacts (that were too large to be well corrected with the motion correction, $\mathrm{n}=2$ ) biasing the quantitative assessment.

\section{Data analysis}

Texture analysis. For each patient, the entire tumor volume was manually segmented by the same senior radiologist using OleaSphere ${ }^{\circledR}$, slice-by-slice, on the last phase of the DCE-MRI acquisition (because the tumor demonstrated the best contrast with surrounding tissues compared with the other phases) and with the help of the conventional sequences acquired during the same MRI examination, i.e.: axial turbo spin echo (TSE) T2-weighted imaging (refocusing angle $=150^{\circ}$, repetition time $=$ $6860 \mathrm{~ms}$, echo time $=120 \mathrm{~ms}$, slice thickness $=4 \mathrm{~mm}$, field-of-view $=250 \times 250 \mathrm{~mm}$ ), coronal or sagittal TSE short time inversion recovery T2-weighted imaging (refocusing angle $=150^{\circ}$, repetition time $=4150 \mathrm{~ms}$, echo time $=69 \mathrm{~ms}$, inversion time $=150 \mathrm{~ms}$, slice thickness $=4 \mathrm{~mm}$, field-of-view $=350 \times 350 \mathrm{~mm})$, axial TSE T1weighted imaging (refocusing angle $=150^{\circ}$, repetition time $=420 \mathrm{~ms}$, echo time $=$ $10 \mathrm{~ms}$, slice thickness $=4 \mathrm{~mm}$, field-of-view $=250 \times 250 \mathrm{~mm}$ ), pre- and post-contrast agent injection axial Fat-Sat TSE T1-weighted imaging (refocusing angle $=180^{\circ}$, repetition time $=581 \mathrm{~ms}$, echo time $=10 \mathrm{~ms}$, slice thickness $=4 \mathrm{~mm}$, field-of-view $=$ $250 \times 250 \mathrm{~mm}$ ). The voxels located at the extreme edge of the tumor were excluded to avoid partial volume effect. The volume of interest (VOI) was then propagated on all the parametric maps ( $\mathrm{K}^{\text {trans }}$ and AUC for the 7 samplings, $\mathrm{K}^{\text {trans }}$ for the 3 truncations). 
Texture analysis consisted in the 3D extraction of 32 quantitative features, provided in Table 1, from histogram analysis (: first order statistics, FOS), grey-level cooccurrence matrix (GLCM), grey-level run-length matrix (GLRM) and grey-level size zone matrix (GLSZM) (32-34). Before, $\mathrm{K}^{\text {trans }}$ and AUC values were discretized in 256 bins. The spatial offset was fixed to a displacement of 4 pixels and an angle of $45^{\circ}$ (details regarding the definitions and calculations of the texture features are given in Supplementary Data 1). No technique for resampling or standardization was applied because the voxel size was initially the same for all patients and texture analysis was performed on scaled parametric maps and not on raw MRI data.

For each patient 'p' $\left(\mathrm{i} \in\left\{1 ; 2 ; \ldots ; \mathrm{N}_{\mathrm{p}}\right\}\right.$ where $\mathrm{N}_{\mathrm{p}}$ is the total number of included patients), for each feature ' $\mathrm{F}_{\mathrm{i}}$ ' $(\mathrm{i} \in\{1 ; 2 ; \ldots ; 32\})$ and for each DCE-MRI map ' $\mathrm{X}(\mathrm{X} \in$ $\left.\left\{\mathrm{K}^{\text {trans; }} \mathrm{AUC}\right\}\right)$, we extracted 7 paired values of $\mathrm{F}_{\mathrm{p}, \mathrm{i}, \mathrm{X}}$ regarding sampling and 3 paired values of $\mathrm{F}_{\mathrm{p}, \mathrm{i}, \mathrm{X}}$ regarding scan duration $\left(\mathrm{F}_{\mathrm{p}, \mathrm{i}, \mathrm{X}}(\mathrm{dt})\right.$ and $\mathrm{F}_{\mathrm{p}, \mathrm{X} X}(\mathrm{~T})$, respectively).

Coefficient of variation $(\mathrm{CV})$. We calculated the mean $\mathrm{CV}$ and its standard deviation (SD) on each feature $F_{i}$ in order to assess the influence of sampling (: CV-dt, with AUC or $\left.\mathrm{K}^{\text {trans }}\right)$, and scan duration (: $\mathrm{CV}-\mathrm{T}$, with $\mathrm{K}^{\text {trans }}$ ) on the dispersion of the texture features, as follows:

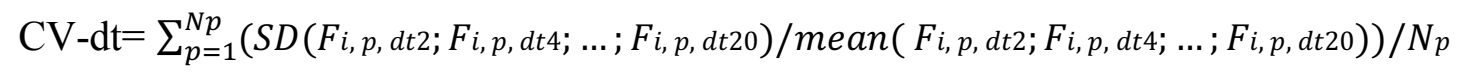

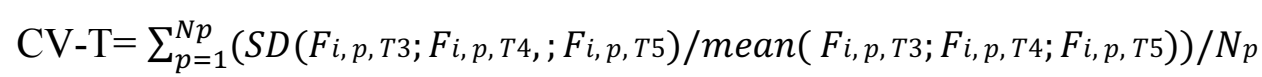

We defined a texture feature as very poorly variable if $\mathrm{CV}<0.1$, poorly variable if $\mathrm{CV}$ $\in[0.1-0.2[$, mildly variable if $\mathrm{CV} \in[0.2-0.5[$, highly variable if $\mathrm{CV} \in[0.5-1[$ and extremely variable if $\mathrm{CV} \geq 1.0$.

\section{Statistical analysis}

Statistical analyses were performed using SPSS (IBM corp, version 21.0, Armonk, NY) and GraphPad Prism (GraphPad Software, version 7, San Diego, CA). Variables were expressed as average, standard deviation, median, and range, as appropriate. A p-value $<0.05$ was deemed significant. All tests were two-tailed.

Normality was assessed for each continuous value by using the Shapiro-Wilk test. Repeated-measures (rm-) ANOVA with Geisser-Greenhouse correction for nonsphericity and post-hoc Tukey tests with corrections for multiple comparisons using statistical hypothesis testing (or non-parametric equivalent rm-Friedman tests with 
post-hoc Dunn tests) were used to study the influence of sampling and truncating on texture features extracted from $\mathrm{K}^{\text {trans }}$ and AUC maps.

For texture features with a statistical influence of temporal resolution, a linear model was applied for correlation analysis. A Bonferroni correction was applied for multiple comparisons and a p-value of 0.01 was considered significant.

Finally, we investigated the influence of temporal parameters on the performance of a model aiming at predicting the treatment response based on texture features from $\mathrm{K}^{\text {trans }}$ and AUC maps. Twenty patients were analyzable for this part of the study; the 5 others were either operated directly after the MRI without chemotherapy or were not sufficiently followed-up. Texture features that were associated with the response at univariate level according to Student t-tests (or Mann-Whitney tests) with a p-value $<0.05$ were entered in a multivariate binary logistic regression using a backward stepwise selection method based on the probability of the Wald statistics. The area under the receiver operating characteristics curve (AUROC) of the models were calculated according to scan durations and temporal resolutions and compared according to the Delong methods (35). 


\section{RESULTS}

\section{Patients (Table 2)}

Twenty-five patients were finally included (9/25 (36\%) women, median age 68 years old, range: 31-94). The most frequent histotype was undifferentiated pleomorphic sarcoma. The median size was $81 \mathrm{~mm}$ (range: 42-180) and the median tumor volume was $0.357 \mathrm{~L}$ (range: $0.023-1.048$ ).

\section{Influence of temporal parameters on DCE-MRI texture features}

Table 3 provides the results of the rm-ANOVA (or rm-Friedman test). Regarding the effect of temporal resolution, 12/32 (37.5\%) AUC-based texture features were significantly influenced: 5/7 FOS features, 1/9 GLCM features, 2/9 GLRLM features and 4/7 GLSZM features (range of p-value $=0.0395$ to $<0.0001$ ). Fourteen out of 32 $(43.8 \%) \mathrm{K}^{\text {trans}}$-based texture features were significantly influenced by temporal resolution: 1/7 FOS features, 5/9 GLCM features, 4/9 GLRLM features and 4/7 GLSZM features (range of p-value $=0.0331$ to 0.0007 ).

Regarding the effect of scan duration, $23 / 32$ (71.9\%) $\mathrm{K}^{\text {trans }}$-based texture features were significantly influenced: 7/7 FOS features, 8/9 GLCM features, 6/9 GLRLM features and 2/7 GLSZM features (range of $\mathrm{p}$-value $=0.0321$ to $<0.0001$ ).

Three texture features were influenced by temporal parameters in the 3 settings: GLRLM_GL_Variance, GLSZM_Large_area_emphasis, and GLSZM_Zone_variance. Six texture features were not, no matter the setting: GLCM_Correlation, GLRLM_Gray_level_non-uniformity, GLRLM_Run_entropy, GLRLM_Run_variance, GLSZM_Small_area_emphasis and GLSZM_Zone_entropy. A summary of post-hoc tests is given in Table 4. Regarding the influence of temporal resolution on AUC maps, the highest number of texture features that statistically differed was observed in post-hoc comparisons between $\mathrm{dt}=20 \mathrm{~s}$ and $\mathrm{dt}=2 \mathrm{~s}(10 / 32$, $31.3 \%)$ and $\mathrm{dt}=20 \mathrm{~s}$ and $\mathrm{dt}=4 \mathrm{~s}(9 / 32,28.1 \%)$. On $\mathrm{K}^{\text {trans }}$ maps, the highest differences were seen between $\mathrm{dt}=20 \mathrm{~s}$ and $\mathrm{dt}=2 \mathrm{~s}(10 / 32,59.4 \%)$, followed by $\mathrm{dt}=20 \mathrm{~s}$ versus $\mathrm{dt}=6 \mathrm{~s}$ (6/32, 18.8\%). Regarding the influence of truncating on $\mathrm{K}^{\text {trans }}$ maps, 23/32 $(71.9 \%)$ and $17 / 32(53.1 \%)$ texture features were significantly different between $\mathrm{T}=5 \mathrm{~min}$ and $\mathrm{T}=4 \mathrm{~min}$, and between $\mathrm{T}=5 \mathrm{~min}$ and $\mathrm{T}=3 \mathrm{~min}$, respectively.

Significant linear correlations were found between sampling on AUC and

GLSZM_Size_zone_non-uniformity $\quad\left(\mathrm{p}=0.0018, \quad \mathrm{r}^{2}=0.879\right) \quad$ and GLSZM_Zone_variance $\left(p=0.0043, r^{2}=0.830\right)$. Similarly, there were significant linear 
correlations between sampling on $\mathrm{K}^{\text {trans }}$ and 4 texture features: GLSZM_nonuniformity $\left(\mathrm{p}<0.0001, \mathrm{r}^{2}=0.978\right)$, GLSZM_Large area emphasis $\left(\mathrm{p}=0.0009, \mathrm{r}^{2}=0.909\right)$, GLSZM_Size_zone_non-uniformity $\left(\mathrm{p}=0.0002, \mathrm{r}^{2}=0.954\right)$, GLSZM_Zone_variance $\left(p=0.0002, r^{2}=0.950\right)$ (Fig. 2). Variations in scan duration did not reveal any linear correlations. Details for linear regressions are given in Supplementary Data 2. Figure 3 shows an example of variation of parametric maps and corresponding histograms with changes in scan duration and temporal resolution.

\section{Effect of temporal parameters on the dispersion of DCE-MRI texture features}

Figure 4 and Table 5 summarize the analysis of dispersion of texture features according to the 3 configurations (i.e. AUC-sampling, Ktrans-sampling, Ktranstruncating). Most texture features extracted from AUC maps remained poorly to mildly variable with changes in sampling. Only GLCM_Cluster_prominence was categorized as highly variable $(\mathrm{CV}-\mathrm{dt}=0.50)$, while 11 out of $32(34.4 \%)$ texture features extracted from $\mathrm{K}^{\text {trans }}$ were highly to extremely variable (range of $\mathrm{CV}-\mathrm{dt}=0.50$ 1.11).

Five texture features extracted from $\mathrm{K}^{\text {trans }}$ maps were highly variable with changes in scan duration: FOS_Average, FOS_Energy, FOS_Inter-quartile_range, FOS_Standard_deviation and GLSZM_Zone_entropy (range of CV-T=0.54-0.96). Supplementary Data 3 provides all the values of CV with SD.

\section{Effect of temporal parameters on a prediction based on DCE-MRI texture features}

Twenty patients were treated with chemotherapy and were analyzable for this sub-part of the study. There were 5/20 (25\%) good responses (4 good histological responses and 1 partial response after 6 months without changing treatment). Table 6 shows the results of univariate analyses and performances of the models quantified by AUROC. After univariate analyses, the selected variables entered in the binary logistic regression were not systematically the same when temporal resolution and scan duration changed, even if some were frequently encountered: AUC_GLRLM_Run_entropy (in 8 models), Ktrans_GLSZM_Small_area_emphasis (in 3 models). AUROC ranged from 0.77 (CI95\%=(0.54-1.00)) with a scan duration of $5 \mathrm{~min}$ and a temporal resolution of $6 \mathrm{~s}$, to $0.90(\mathrm{CI} 95 \%=(0.74-1.00))$ with a scan duration of $4 \mathrm{~min}$ and a temporal resolution of $6 \mathrm{~min}$. Details of the univariate analyses 
for each model and comparisons between AUROC can be found in Supplementary Data 4 and Supplementary Data 5, respectively. 


\section{DISCUSSION}

In this study, we examined the influence of temporal parameters on a large set of widely used and easily available statistical texture features extracted from whole tumor volumes segmented on $\mathrm{K}^{\text {trans }}$ and AUC parametric maps. We found that a large number of them were significantly dependent on the scan duration and temporal resolution. Moreover, merely half of them remained poorly variable with changes in temporal parameters. This led to non-negligible variations in the AUROCs of the models for a response prediction to chemotherapy, even though the comparisons did not provide statistical differences.

A major challenge for radiomics approaches is to ensure a good quality of the quantitative data on which they rely. Besides reproducibility, repeatability, nonredundancy and validity, quality here means controlling the bias due to slight variations in the imaging acquisition parameters (2). Standardizing the imaging protocols between radiological centers is unavoidable. However, thousands of MRI examinations (and DCE-MRI sequences) have already been acquired and the temptation to pool data from different MR-systems in order to begin to build and test predictive models for key oncological questions is understandable. Our study focused on STS as a tumor model to examine the influence of temporal parameters. Highgrade STS are characterized by complex architectures and changes during treatments, making them particularly appropriate for radiomics. In particular, Vallières et al. stressed the influence of the post-processing parameters of imaging on a prediction model of occurrence of lung metastases in STS patients (36).

Our results are in agreement with previous studies that investigated the influence of temporal parameters on dynamic acquisitions. Othman et al. showed that shorter scan duration was responsible for overestimation of pharmacokinetic parameters and lead to incorrect classifications of benign prostate lesions as malignant (24). A similar influence on prediction models was found with breast lesions (26). This could be explained by the Tofts model itself because it assumes an immediate equilibrium between the compartments though it requires up to $2 \mathrm{~min}$ in case of breast imaging (37). Poor temporal resolutions can also lead to incorrect assessments of pharmacokinetic parameters according to pre-clinical and clinical studies regarding prostate lesions - even if it did not significantly modify the ability of these parameters to discriminate benign and malignant tumors (25). Heisen et al. showed that the $\mathrm{K}^{\text {trans }}$ 
variations could go up to $25 \%$ of its initial value with varying temporal resolutions (38). In the single study that focused on the acquisition parameters at risk of influencing perfusion maps, Bogowicz et al. found that the percentage of unstable texture features ranged from 56 to $98 \%$ with different artery contouring, and from 58 to $75 \%$ with different temporal resolution, which is higher than in our study (27). This highest variability in their study may be explained by the difference in histological types, in imaging modality (CT vs. MRI), in the texture features that were calculated and in the perfusion maps that were studied (blood flow, mean time transit and blood volume vs. AUC and $\mathrm{K}^{\text {trans }}$ in our studies).

Regarding STS, our results also highlight potential cut-offs for temporal resolution and scan duration beyond which statistical differences in texture features from DCEMRI maps may occur. Indeed, even if temporal resolution had an influence on several texture features, post-hoc tests with correction for multiple comparisons showed that data with temporal resolution below 8 s could be pooled, as well as data with temporal resolution above 8s. On the contrary, we found that data obtained with a scan duration of $5 \mathrm{~min}$ should not be pooled with those with a scan duration of 3 or $4 \mathrm{~min}$.

After correction of multiple tests, some radiomics features extracted from AUC and $\mathrm{K}^{\text {trans }}$ maps demonstrated linear relationships with sampling. All belonged to the GLSZM category. GLSZM_Size_zone_non_uniformity and GLSZM_Grey_level_non_uniformity increased with downsampling, suggesting more heterogeneity in intensity values and in size zone volumes of the same grey level voxels with low temporal resolutions (high dt). Conversely, GLSZM_Large_area_emphasis and GLSZM_Zone_variance tended to decrease, suggesting that poor temporal resolution (high $\mathrm{dt}$ ) led to smaller size zones. No linear correlation was found with truncating, probably because we only generated 3 time points. Poor temporal resolution (high dt) can be responsible for less accurate estimations, especially when the tumor - or areas in the tumor-demonstrates a rapid and strong enhancement (wash-in and peak on time-intensity curves). Consequently, hypervascularized intra-tumoral areas with high $\mathrm{K}^{\text {trans }}$ and AUC values could be missed, resulting in areas with lower values of $\mathrm{K}^{\text {trans }}$ and AUC.

All the categories of texture features seemed equally influenced by temporal resolution, but the dispersion was more marked with FOS, then GLCM, GLRLM and finally GLSZM. The high sensitivity of FOS could be due to their lack of spatial information. Indeed, all the voxels are pooled without considering the fact that 
adjacent voxels may react similarly and proportionally to changes in temporal parameters, which is the case of GLCM, GLSZM and GLRLM.

Regarding the influence of temporal parameters on the performance of a model for response prediction based on AUC and $\mathrm{K}^{\text {trans }}$ of STS, we identified 6 relevant features that were selected across the different models, namely: FOS_Inter_quartile_range, FOS_Standard_deviation, GLCM_Correlation, GLRLM_Long_run_emphasis, GLRLM_Run_entropy and GLSZM_Small_area_emphasis. Interestingly, GLRLM_Run_entropy and GLRLM_Small_area_emphasis were not significantly influenced by temporal resolution and showed low CVs. Thus, these texture features could be good candidates for multi-center studies based on DCE-MRI sequences of STS. However, these results do not mean that ultra-fast DCE-MRI acquisitions are unnecessary. The aim of DCE-MRI is to represent and estimate at best the vascular characteristics of tumors. Hypervascularized tumors require excellent temporal resolutions. If some researchers want to increase the statistical power of their radiomics study by putting together DCE-MRIs with different temporal parameters, then it should be carefully done because it could introduce a significant bias in their results.

We did not investigate the test-retest reproducibility of DCE-MRI-based texture features because it was hardly justifiable to inject a contrast agent twice in cancer patients. However, further radiomics studies performed on perfusion phantoms could help analyzing this crucial aspect. It should be noted that we designed our study to limit bias that could have been introduced in the quantification of heterogeneity: we utilized the same 1.5T MR-system, the same DCE-MRI sequences with the same acquisition parameters following $\mathrm{QIBA}{ }^{\circledR}$ recommendations, the same contrast agent, the same imaging filters, the same software for post-processing and the same feature extraction parameters. Nevertheless, we had to adjust the coils because of variations in tumor sizes and locations, which may have introduced some bias. Moreover, truncating and downsampling the AIFs probably contributed to the variations in texture features from DCE-MRI parametric maps, even if we kept the same voxels in the same artery of interest. Indeed, the AIF is crucial in pharmacokinetic modeling (31). For instance, a recent study showed that $\mathrm{K}^{\text {trans }}$ could range from $0.25 / \mathrm{min}$ to more $2 / \mathrm{min}$ in prostate cancers in a same series of DCE-MRI acquisitions depending on the method to determine the AIF from different cancer centers, which led to variations in times to peak and peak amplitudes (39). Herein, poor temporal 
resolutions could lead to missing the real AIF peak though the $\mathrm{K}^{\text {trans }}$ estimation strongly relies on it, as well as to superposition of the AIF and the tumor enhancement curve during the first part of the acquisition.

Our study has several limits. First, the population was small and made of heterogeneous histological types, though all were high-grade. Some tumors enhanced rather homogeneously and progressively (for instance myxoid/round cell liposarcomas) while others showed more heterogeneous enhancements, with the timeintensity curves of some components displaying a strong wash-in, followed by a peak and a wash-out (for instance, undifferentiated pleomorphic sarcomas). Temporal parameters were certainly more influential in the second case. Nevertheless, until now, the inclusion of STS patients in clinical trials relies on tumor grade and not on histotypes. Second, we did not investigate other semi-quantitative and pharmacokinetic DCE-MRI parameters (such as Time-to-peak, Wash-in, Wash-out, $\mathrm{K}^{\mathrm{ep}}$ [efflux rate constant from the extra-cellular, extra-vascular space to the plasma compartment], $\mathrm{K}^{\mathrm{el}}$ [contrast agent elimination rate constant], $\mathrm{V}_{\mathrm{e}}$ [extra-cellular, extravascular space volume], $\mathrm{V}_{\mathrm{p}}$ [plasmatic volume]), or other perfusion models than extended Tofts. We decided to focus on the most studied parameters in the literature. However, given that some areas in STS can show a wash-out, it is also possible that the pharmacokinetics that quantify the decreasing part of the time-intensity curve are also influenced by temporal parameters. Third, alternative methods for downsampling could have been applied by recombining k-space data instead of removing some phases (38). In a clinical setting, there is a compromise between temporal resolution, signal-to-noise ratio and spatial resolution. A decrease in temporal resolution will benefit signal-to-noise-ratio (by averaging twice if there is a down-sampling by a factor 2, for instance). Fourth, our multivariate models for the outcome sub-study can be questioned since only 20 patients were included with 2 definitions of good treatment responses depending on the patient's operability. We used a classical statistical approach without validation cohort to build the predictive models. More advanced selection methods could have been used (for instance: least absolute shrinkage and selection operator, ElasticNet, supervised principal component analysis), as well as supervised machine-learning classifiers (for instance: random forest, k-nearest neighbors, support vector machines) or deep learning (40). 
Consequently, we did not test if the influence of temporal parameters was still present with these other statistical methods. The aim of this part of the study was to illustrate the influence of temporal parameters on a prediction and not to validate a radiomics model for response prediction. However, we hope that further prospective studies will take into account this research aiming at improving the quality of radiomics methods in order to build clean and robust models to answer key oncological questions.

To conclude, our study screened several aspects of the influence of temporal parameters on texture features extracted from DCE-MRI parametric maps of STS. We showed that both scan duration and temporal resolution introduced a non-negligible variability in the quantification of heterogeneity that could lead to a decreased performance of prediction models for response to chemotherapy. In addition to all the other acquisition and post-processing parameters, standardizing the scan duration and temporal resolution of DCE-MRI must be considered in prospective multi-centric trials to build reliable radiomics approaches. 


\section{REFERENCES}

1. O’Connor JPB: Cancer heterogeneity and imaging. Semin Cell Dev Biol 2017; 64:48-57.

2. Limkin EJ, Sun R, Dercle L, et al.: Promises and challenges for the implementation of computational medical imaging (radiomics) in oncology. Ann Oncol 2017; 28:1191-1206.

3. Jackson A, O'Connor JPB, Parker GJM, Jayson GC: Imaging tumor vascular heterogeneity and angiogenesis using dynamic contrast-enhanced magnetic resonance imaging. Clin Cancer Res 2007; 13:3449-3459.

4. O'Connor JPB, Aboagye EO, Adams JE, et al.: Imaging biomarker roadmap for cancer studies. Nat Rev Clin Oncol 2017; 14:169-186.

5. Ginsburg SB, Algohary A, Pahwa S, et al.: Radiomic features for prostate cancer detection on MRI differ between the transition and peripheral zones: Preliminary findings from a multi-institutional study. J Magn Reson Imaging 2017; 46:184-193.

6. Nie K, Shi L, Chen Q, et al.: Rectal Cancer: Assessment of Neoadjuvant Chemoradiation Outcome based on Radiomics of Multiparametric MRI. Clin Cancer Res 2016; 22:5256-5264.

7. Braman NM, Etesami M, Prasanna P, et al.: Intratumoral and peritumoral radiomics for the pretreatment prediction of pathological complete response to neoadjuvant chemotherapy based on breast DCE-MRI. Breast Cancer Res 2017; 19:57.

8. Bowen SR, Yuh WTC, Hippe DS, et al.: Tumor radiomic heterogeneity: Multiparametric functional imaging to characterize variability and predict response following cervical cancer radiation therapy. J Magn Reson Imaging 2018; 47:13881396.

9. Liu C, Ding J, Spuhler K, et al.: Preoperative prediction of sentinel lymph node metastasis in breast cancer by radiomic signatures from dynamic contrast-enhanced MRI. J Magn Reson Imaging 2019; 49:131-140.

10. Thomassin-Naggara I, Soualhi N, Balvay D, Darai E, Cuenod C-A: Quantifying tumor vascular heterogeneity with DCE-MRI in complex adnexal masses: A preliminary study. J Magn Reson Imaging 2017; 46:1776-1785.

11. Fan M, Cheng H, Zhang P, et al.: DCE-MRI texture analysis with tumor subregion partitioning for predicting Ki-67 status of estrogen receptor-positive breast cancers. J Magn Reson Imaging 2018; 48:237-247.

12. Rose CJ, Mills SJ, O'Connor JPB, et al.: Quantifying spatial heterogeneity in dynamic contrast-enhanced MRI parameter maps. Magn Reson Med 2009; 62:488499.

13. Zhao F, Ahlawat S, Farahani SJ, et al.: Can MR imaging be used to predict tumor grade in soft-tissue sarcoma? Radiology 2014; 272:192-201.

14. Soldatos T, Ahlawat S, Montgomery E, Chalian M, Jacobs MA, Fayad LM: Multiparametric Assessment of Treatment Response in High-Grade Soft-Tissue Sarcomas with Anatomic and Functional MR Imaging Sequences. Radiology 2016; 278:831-840.

15. Meyer JM, Perlewitz KS, Hayden JB, et al.: Phase I trial of preoperative chemoradiation plus sorafenib for high-risk extremity soft tissue sarcomas with dynamic contrast-enhanced MRI correlates. Clin Cancer Res 2013; 19:6902-6911. 16. Crombé A, Le Loarer F, Cornelis F, et al.: High-grade soft-tissue sarcoma: optimizing injection improves MRI evaluation of tumor response. Eur Radiol 2019; 29:545-555. 
17. Huang W, Beckett BR, Tudorica A, et al.: Evaluation of Soft Tissue Sarcoma Response to Preoperative Chemoradiotherapy Using Dynamic Contrast-Enhanced Magnetic Resonance Imaging. Tomography 2016; 2:308-316.

18. Tian F, Hayano K, Kambadakone AR, Sahani DV: Response assessment to neoadjuvant therapy in soft tissue sarcomas: using CT texture analysis in comparison to tumor size, density, and perfusion. Abdom Imaging 2015; 40:1705-1712.

19. Hayano K, Tian F, Kambadakone AR, et al.: Texture Analysis of Non-ContrastEnhanced Computed Tomography for Assessing Angiogenesis and Survival of Soft Tissue Sarcoma. J Comput Assist Tomogr 2015; 39:607-612.

20. Buch K, Kuno H, Qureshi MM, Li B, Sakai O: Quantitative variations in texture analysis features dependent on MRI scanning parameters: A phantom model. J Appl Clin Med Phys 2018; 19:253-264.

21. Mayerhoefer ME, Szomolanyi P, Jirak D, Materka A, Trattnig S: Effects of MRI acquisition parameter variations and protocol heterogeneity on the results of texture analysis and pattern discrimination: an application-oriented study. Med Phys 2009; 36:1236-1243.

22. Collewet G, Strzelecki M, Mariette F: Influence of MRI acquisition protocols and image intensity normalization methods on texture classification. Magn Reson Imaging 2004; 22:81-91.

23. Ford J, Dogan N, Young L, Yang F: Quantitative Radiomics: Impact of Pulse Sequence Parameter Selection on MRI-Based Textural Features of the Brain. Contrast Media Mol Imaging 2018; 2018:1729071.

24. Othman AE, Falkner F, Martirosian P, et al.: Optimized Fast Dynamic ContrastEnhanced Magnetic Resonance Imaging of the Prostate: Effect of Sampling Duration on Pharmacokinetic Parameters. Invest Radiol 2016; 51:106-112.

25. Othman AE, Falkner F, Weiss J, et al.: Effect of Temporal Resolution on Diagnostic Performance of Dynamic Contrast-Enhanced Magnetic Resonance Imaging of the Prostate. Invest Radiol 2016; 51:290-296.

26. Hao W, Zhao B, Wang G, Wang C, Liu H: Influence of scan duration on the estimation of pharmacokinetic parameters for breast lesions: a study based on CAIPIRINHA-Dixon-TWIST-VIBE technique. Eur Radiol 2015; 25:1162-1171.

27. Bogowicz M, Riesterer O, Bundschuh RA, et al.: Stability of radiomic features in CT perfusion maps. Phys Med Biol 2016; 61:8736-8749.

28. Cousin S, Crombe A, Stoeckle E, et al.: Clinical, radiological and genetic features, associated with the histopathologic response to neoadjuvant chemotherapy (NAC) and outcomes in locally advanced soft tissue sarcoma (STS) patients (pts). JCO 2017; 35(15_suppl):11014-11014.

29. Ray-Coquard I, Collard O, Ducimetiere F, et al.: Treatment patterns and survival in an exhaustive French cohort of pazopanib-eligible patients with metastatic soft tissue sarcoma (STS). BMC Cancer 2017; 17:111.

30. Michaely HJ, Morelli JN, Budjan J, et al.: CAIPIRINHA-Dixon-TWIST (CDT)volume-interpolated breath-hold examination (VIBE): a new technique for fast timeresolved dynamic 3-dimensional imaging of the abdomen with high spatial resolution. Invest Radiol 2013; 48:590-597.

31. Tofts PS, Brix G, Buckley DL, et al.: Estimating kinetic parameters from dynamic contrast-enhanced T(1)-weighted MRI of a diffusable tracer: standardized quantities and symbols. J Magn Reson Imaging 1999; 10:223-232.

32. Haralick RM, Shanmugam K, Dinstein I: Textural features of image classification. IEEE Transactions on Systems, Man, \& Cybernetics 1973; 3:610-621. 
33. Galloway MM: Texture analysis using gray level run lengths. Computer Graphics and Image Processing 1975; 4:172-179.

34. Thibault G, Fertil B, Navarro C, et al.: Texture Indexes and Gray Level Size Zone Matrix Application to Cell Nuclei Classification. 2009.

35. DeLong ER, DeLong DM, Clarke-Pearson DL: Comparing the areas under two or more correlated receiver operating characteristic curves: a nonparametric approach. Biometrics 1988; 44:837-845.

36. Vallières M, Freeman CR, Skamene SR, El Naqa I: A radiomics model from joint FDG-PET and MRI texture features for the prediction of lung metastases in softtissue sarcomas of the extremities. Phys Med Biol 2015; 60:5471-5496.

37. Kuhl CK, Mielcareck P, Klaschik S, et al.: Dynamic breast MR imaging: are signal intensity time course data useful for differential diagnosis of enhancing lesions? Radiology 1999; 211:101-110.

38. Heisen M, Fan X, Buurman J, van Riel NAW, Karczmar GS, ter Haar Romeny

BM: The influence of temporal resolution in determining pharmacokinetic parameters from DCE-MRI data. Magn Reson Med 2010; 63:811-816.

39. Huang W, Chen Y, Fedorov A, et al.: The Impact of Arterial Input Function Determination Variations on Prostate Dynamic Contrast-Enhanced Magnetic Resonance Imaging Pharmacokinetic Modeling: A Multicenter Data Analysis Challenge. Tomography 2016; 2:56-66.

40. Erickson BJ, Korfiatis P, Akkus Z, Kline TL: Machine Learning for Medical Imaging. Radiographics 2017; 37:505-515. 
TABLE 1. MRI texture features extracted from $\mathrm{K}^{\text {trans }}$ and AUC maps.

\begin{tabular}{cccc}
\hline First-order Feature & $\begin{array}{c}\text { Grey level co- } \\
\text { occurrence matrix }\end{array}$ & $\begin{array}{c}\text { Grey level run length } \\
\text { matrix }\end{array}$ & $\begin{array}{c}\text { Grey level size zone } \\
\text { matrix }\end{array}$ \\
\hline Average & Cluster prominence & GL non-uniformity & GL non-uniformity \\
Energy & Cluster shade & GL variance & GL variance \\
Entropy & Cluster tendency & High GL run emphasis & Large area emphasis \\
Inter-quartile range & Contrast & Low GL run emphasis & Small area emphasis \\
Kurtosis & Correlation & Long run emphasis & Size zone non-uniformity \\
Skewness & Inverse difference moment & Run entropy & Zone entropy \\
Standard deviation & Joint average & Run length non-uniformity & Zone variance \\
& Joint energy & Run variance & \\
& Joint entropy & Short run emphasis & \\
& & &
\end{tabular}

NOTE. Abbreviations: GL: grey level 
TABLE 2. Epidemiological features of the population study.

\begin{tabular}{lc}
\hline Characteristics & Patients \\
\hline Gender & $16 / 25(64 \%)$ \\
Men & $9 / 25(36 \%)$ \\
Women & $68(31-94)$ \\
Age (years) & \\
Histological types & $13 / 25(52 \%)$ \\
Undifferentiated pleomorphic sarcoma & $4 / 25(16 \%)$ \\
Myxoid/round cells liposarcoma & $2 / 25(8 \%)$ \\
Rhabdomyosarcoma & $1 / 25(4 \%)$ \\
Myxofibrosarcoma & $2 / 25(8 \%)$ \\
Synovial sarcoma & $1 / 25(4 \%)$ \\
Pleomorphic liposarcoma & $2 / 25(8 \%)$ \\
Undifferentiated sarcoma - others & \\
Location & $4 / 25(16 \%)$ \\
Upper limb & $1 / 25(4 \%)$ \\
Shoulder girdle & $2 / 25(8 \%)$ \\
Trunk wall & $18 / 25(72 \%)$ \\
Lower limb & $81(42-180)$ \\
Size (mm) & $(22816-1048136)$ \\
Number of voxels & $959(0.023-1.048)$ \\
Volume (L) & \\
Tumor depth & $16 / 25(64 \%)$ \\
Deep & $8 / 25(32 \%)$ \\
Deep and superficial & $1 / 25(4 \%)$ \\
Superficial &
\end{tabular}

NOTE. Data are number of patients with percentage in parentheses, except for age, size, number of voxels and volume of the tumor, given as median and range. 
TABLE 3. Assessment of the influence of temporal resolution (sampling) and scan duration (truncating) on texture parameters extracted from DCE-MRI parametric maps ( $\mathrm{K}^{\text {trans }}$ and AUC).

\begin{tabular}{|c|c|c|c|c|c|c|}
\hline \multirow{2}{*}{ Texture features } & \multicolumn{2}{|c|}{ AUC - Sampling } & \multicolumn{2}{|c|}{$\mathrm{K}^{\text {trans }}$ - Sampling } & \multicolumn{2}{|c|}{$\mathrm{K}^{\text {trans }}$ - Truncating } \\
\hline & F-value & p-value & F-value & p-value & F-value & p-value \\
\hline \multicolumn{7}{|l|}{ First-order Feature } \\
\hline Average & 18.54 & $0.0050^{* *}$ & 5.12 & 0.5290 & 14.60 & $0.0022^{\star *}$ \\
\hline Energy & 25.55 & $0.0003^{* * *}$ & 2.13 & 0.9072 & 15.00 & $0.0018^{* *}$ \\
\hline Entropy & 7.15 & 0.3069 & 16.46 & $0.0115^{*}$ & 19.19 & $0.0002^{* * *}$ \\
\hline Inter-quartile range & 8.607 & 0.1969 & 5.61 & 0.4688 & 15.05 & $0.0018^{* *}$ \\
\hline Kurtosis & 50.89 & $<0.0001^{* * *}$ & 6.35 & 0.0958 & 15.49 & $0.0168^{*}$ \\
\hline Skewness & 41.57 & $<0.0001^{* * *}$ & 10.88 & 0.0921 & 8.80 & $0.0321^{*}$ \\
\hline Standard deviation & 26.12 & $0.0002^{* * *}$ & 1.77 & 0.9395 & 14.50 & $0.0023^{* *}$ \\
\hline \multicolumn{7}{|l|}{ GLCM } \\
\hline Cluster prominence & 17.07 & $0.0090^{* *}$ & 11.46 & 0.0751 & 16.20 & $0.0010^{* *}$ \\
\hline Cluster shade & 5.07 & 0.5354 & 12.15 & 0.0587 & 12.55 & $0.0057^{* *}$ \\
\hline Cluster tendency & 10.30 & 0.1127 & 9.96 & 0.1262 & 23.85 & $<0.0001^{* * *}$ \\
\hline Contrast & 9.27 & 0.1589 & 13.7 & $0.0331^{*}$ & 17.85 & $0.0005^{\star * *}$ \\
\hline Correlation & 2.78 & 0.8358 & 5.59 & 0.4709 & 4.25 & 0.2357 \\
\hline Inverse difference moment & 6.58 & 0.3617 & 13.70 & $0.0331^{*}$ & 17.85 & $0.0005^{\star * *}$ \\
\hline Joint average & 1.94 & 0.9253 & 13.96 & $0.0301^{*}$ & 18.75 & $0.0003^{* * *}$ \\
\hline Joint energy & 6.45 & 0.3750 & 13.83 & $0.0316^{*}$ & 18.40 & $0.0004^{* * *}$ \\
\hline Joint entropy & 6.87 & 0.3327 & 16.64 & $0.0233^{*}$ & 19.55 & $0.0002^{* * *}$ \\
\hline \multicolumn{7}{|l|}{ GLRLM } \\
\hline GL non-uniformity & 0.96 & 0.9869 & 5.83 & 0.4427 & 1.05 & 0.7892 \\
\hline GL variance & 20.74 & $0.0020^{* *}$ & 17.7 & $0.0070^{*}$ & 29.30 & $<0.0001^{* * *}$ \\
\hline High GL run emphasis & 5.74 & 0.4525 & 19.16 & $0.0039^{\star *}$ & 29.30 & $<0.0001^{* * *}$ \\
\hline Low GL run emphasis & 3.27 & 0.7746 & 19.16 & $0.0039^{* *}$ & 29.30 & $<0.0001^{* * *}$ \\
\hline Long run emphasis & 11.00 & 0.0884 & 17.05 & $0.0091^{* *}$ & 31.50 & $<0.0001^{* * *}$ \\
\hline Run entropy & 0.32 & $0.8632 \S$ & 1.40 & $0.2556^{\S}$ & 0.9251 & $0.4056^{\S}$ \\
\hline Run length non-uniformity & 7.32 & 0.2918 & 11.46 & 0.0753 & 14.85 & $0.0019^{* *}$ \\
\hline Run variance & 10.47 & 0.1062 & 11.36 & 0.0778 & 5.95 & 0.1141 \\
\hline Short run emphasis & 13.23 & $0.0395^{*}$ & 1.56 & 0.2133 & 22.95 & $<0.0001^{* * *}$ \\
\hline \multicolumn{7}{|l|}{ GLSZM } \\
\hline GL non-uniformity & 9.69 & 0.1384 & 15.31 & $0.0180^{*}$ & 10.94 & 0.012 \\
\hline GL variance & 25.72 & $0.0003^{* * *}$ & 11.89 & 0.0645 & 10.18 & 0.0171 \\
\hline Large area emphasis & 19.29 & $0.0037^{* *}$ & 21.41 & $0.0015^{\star *}$ & 15.30 & $0.0016^{\star *}$ \\
\hline Small area emphasis & 9.16 & 0.1649 & 4.91 & 0.5555 & 4.40 & 0.2214 \\
\hline Size zone non-uniformity & 16.11 & $0.0132^{*}$ & 16.87 & $0.0098^{*}$ & 5.87 & 0.118 \\
\hline Zone entropy & 3.08 & $0.2190^{\S}$ & 4.746 & 0.5768 & 1.01 & $0.3797 \S$ \\
\hline Zone variance & 20.26 & $0.0025^{\star *}$ & 23.46 & $0.0007^{* * *}$ & 15.30 & $0.0016^{* *}$ \\
\hline
\end{tabular}

NOTE. Data are F-values and p-values for the repeated measures (rm-) ANOVA ( : §) or non parametric equivalent rm-Friedman test.

Abbreviations: GL : grey-level; GLCM : grey-level occurrence matrix; GLRLM: grey-level run length matrix; GLSZM: grey level size zone matrix

*: $\mathrm{p} \leq 0.05 ; * *: \mathrm{p}<0.005, * * *: \mathrm{p}<0.001$ 
TABLE 4. Summary of the post-hoc tests: number of texture features that were significantly different when comparing 2 distinct temporal resolution (: sampling) for $\operatorname{AUC}(\mathbf{a})$ and $\mathrm{K}^{\text {trans }}(\mathbf{b})$, or 2 distinct scan durations (: truncating) for $\mathrm{K}^{\text {trans }}(\mathbf{c})$.

\section{a. AUC - Sampling}

\begin{tabular}{|c|c|c|c|c|c|c|c|}
\hline & $d t 2$ & $d t 4$ & $d t 6$ & $d t 8$ & $d t 10$ & $d t 12$ & $d t 20$ \\
\hline$d t 2$ & - & 0 & 0 & 2 & 4 & 1 & 10 \\
\hline$d t 4$ & 0 & - & 0 & 2 & 2 & 0 & 9 \\
\hline$d t 6$ & 0 & 0 & - & 0 & 0 & 0 & 2 \\
\hline$d t 8$ & 2 & 2 & 0 & - & 0 & 0 & 0 \\
\hline$d t 10$ & 4 & 2 & 0 & 0 & - & 0 & 0 \\
\hline$d t 12$ & 1 & 0 & 0 & 0 & 0 & - & 0 \\
\hline$d t 20$ & 10 & 9 & 2 & 0 & 0 & 0 & - \\
\hline
\end{tabular}

\section{b. $\mathrm{K}^{\text {trans }}$ - Sampling}

\begin{tabular}{|c|c|c|c|c|c|c|c|}
\hline & $d t 2$ & $d t 4$ & $d t 6$ & $d t 8$ & $d t 10$ & $d t 12$ & $d t 20$ \\
\hline$d t 2$ & - & 0 & 0 & 0 & 0 & 0 & 10 \\
\hline$d t 4$ & 0 & - & 0 & 0 & 0 & 0 & 2 \\
\hline$d t 6$ & 0 & 0 & - & 0 & 0 & 0 & 6 \\
\hline$d t 8$ & 0 & 0 & 0 & - & 0 & 0 & 0 \\
\hline$d t 10$ & 0 & 0 & 0 & 0 & - & 0 & 0 \\
\hline$d t 12$ & 0 & 0 & 0 & 0 & 0 & - & 0 \\
\hline$d t 20$ & 10 & 2 & 6 & 0 & 0 & 0 & - \\
\hline
\end{tabular}

c. $\mathbf{K}^{\text {trans }}$ - Truncating

\begin{tabular}{|c|c|c|c|}
\hline & T3'00 & T4'00 & T5'00 \\
\hline T3'00 & - & 0 & 17 \\
\hline T4'00 & 0 & - & 23 \\
\hline T5'00 & 17 & 23 & - \\
\hline
\end{tabular}

NOTE. Abbreviations: $d t x$ corresponds to a sampling of ' $x$ ' seconds; Ty corresponds to a scan duration of ' $y$ ' minutes. 
TABLE 5. Degree of dispersion of the texture features from $\mathrm{K}^{\text {trans }}$ and AUC maps according to temporal resolution (: sampling) and scan duration (: truncating).

\begin{tabular}{|c|c|c|c|c|c|}
\hline & $\begin{array}{c}\text { Very poorly variable } \\
\mathrm{CV}<0.10\end{array}$ & $\begin{array}{l}\text { Poorly variable } \\
0.10 \leq \mathrm{CV}<0.20\end{array}$ & $\begin{array}{c}\text { Mildly variable } \\
0.20 \leq \mathrm{CV}<0.50\end{array}$ & $\begin{array}{l}\text { Highly variable } \\
0.50 \leq \mathrm{CV} \leq 1\end{array}$ & $\begin{array}{c}\text { Extremely variable } \\
\text { CV } \geq 1.00\end{array}$ \\
\hline AUC - Sampling & $\begin{array}{c}\text { GLCM_Inverse Difference Moment } \\
\text { GLCM_Joint average } \\
\text { GLCM_Joint energy } \\
\text { GLRLM_GL non-uniformity } \\
\text { GLRLM_High GL run emphasis } \\
\text { GLRLM_Run length non-uniformity } \\
\text { GLRLM_Run entropy } \\
\text { GLRLM_Run variance } \\
\text { GLRLM_Short run emphasis }\end{array}$ & $\begin{array}{c}\text { FOS_Average } \\
\text { FOS_Inter-quartile range } \\
\text { FOS_Standard deviation } \\
\text { GLRLM_Low GL run emphasis } \\
\text { GLRLM_Long run emphasis } \\
\text { GLSZM_Small area emphasis } \\
\text { GLSZM_Zone entropy }\end{array}$ & $\begin{array}{c}\text { FOS_Energy } \\
\text { FOS_Entropy } \\
\text { FOS_Kurtosis } \\
\text { FOS_Skewness } \\
\text { GLCM_Cluster shade } \\
\text { GLCM_Cluster tendency } \\
\text { GLCM_Correlation } \\
\text { GLCM_Contrast } \\
\text { GLCM_Joint entropy } \\
\text { GLRLM_GL variance } \\
\text { GLSZM_GL non-uniformity } \\
\text { GLSZM_GL variance } \\
\text { GLSZM_Large area emphasis } \\
\text { GLSZM_Zone variance } \\
\text { GLSZM_Size zone non-uniformity }\end{array}$ & GLCM_Cluster prominence & \\
\hline $\mathbf{K}^{\text {trans }}$ - Sampling & $\begin{array}{l}\text { GLCM_Inverse Difference Moment } \\
\text { GLCM_Joint average } \\
\text { GLCM_Joint energy } \\
\text { GLRLM_GL non-uniformity } \\
\text { GLRLM_High GL run emphasis } \\
\text { GLRLM_Low GL run emphasis } \\
\text { GLRLM_Run entropy }\end{array}$ & $\begin{array}{l}\text { GLRLM_Run variance } \\
\text { GLRLM_Short run emphasis } \\
\text { GLSZM_Small area emphasis } \\
\text { GLSZM_Zone entropy }\end{array}$ & $\begin{array}{c}\text { FOS_Entropy } \\
\text { GLCM_Correlation } \\
\text { GLRLM_GL variance } \\
\text { GLRLM_Long run emphasis } \\
\text { GLRLM_Run length non-uniformity } \\
\text { GLSZM_GL non-uniformity } \\
\text { GLSZM_GL variance } \\
\text { GLSZM_Large area emphasis } \\
\text { GLSZM_Zone variance } \\
\text { GLSZM_Size zone non-uniformity }\end{array}$ & $\begin{array}{c}\text { FOS_Inter-quartile range } \\
\text { FOS_Kurtosis } \\
\text { FOS_Average } \\
\text { FOS_Skewness } \\
\text { FOS_Standard deviation } \\
\text { GLCM_Cluster prominence } \\
\text { GLCM_Cluster shade } \\
\text { GLCM_Cluster tendency } \\
\text { GLCM_Contrast } \\
\text { GLCM_Joint entropy }\end{array}$ & FOS_Energy \\
\hline $\mathrm{K}^{\text {trans }}$ - Truncating & $\begin{array}{c}\text { GLCM_Inverse Difference Moment } \\
\text { GLCM_Joint average } \\
\text { GLCM_Joint energy } \\
\text { GLRLM_GL non-uniformitY } \\
\text { GLRLM_High GL run emphasis } \\
\text { GLRLM_Low GL run emphasis } \\
\text { GLRLM_Run entropy } \\
\text { GLRLM_Short run emphasis }\end{array}$ & $\begin{array}{c}\text { GLRLM_Long run emphasis } \\
\text { GLRLM_Run length non-uniformity } \\
\text { GLRLM_Run variance } \\
\text { GLSZM_Small area emphasis }\end{array}$ & $\begin{array}{c}\text { FOS_Entropy } \\
\text { FOS_Kurtosis } \\
\text { FOS_Skewness } \\
\text { GLCM_Cluster prominence } \\
\text { GLCM_Cluster shade } \\
\text { GLCM_Cluster tendency } \\
\text { GLCM_Correlation } \\
\text { GLCM_Contrast } \\
\text { GLCM_Joint entropy } \\
\text { GLRLM_GL variance } \\
\text { GLSZM_GL non-uniformity } \\
\text { GLSZM_GL variance } \\
\text { GLSZM_Large area emphasis } \\
\text { GLSZM_Zone variance } \\
\text { GLSZM_Size zone non-uniformity }\end{array}$ & $\begin{array}{c}\text { FOS_Average } \\
\text { FOS_Energy } \\
\text { FOS_Inter-quartile range } \\
\text { FOS_Standard deviation } \\
\text { GLSZM_Zone entropy }\end{array}$ & \\
\hline
\end{tabular}

NOTE. Abbreviations: CV: coefficient of variation; FOS: First order statistics; GL: grey level; GLCM: grey level co-occurrence matrix; GLRLM: grey level run length matrix; GLSZM: grey level size zone matrix 
TABLE 6. Area under the ROC curves of models for response prediction to chemotherapy based on texture features extracted from AUC and $\mathrm{K}^{\text {trans }}$ maps with varying temporal resolution (sampling) and scan duration (truncating)

\begin{tabular}{|c|c|c|c|c|}
\hline \multirow{2}{*}{$\begin{array}{l}\text { Temporal } \\
\text { parameters }\end{array}$} & \multicolumn{2}{|l|}{ Univariate analysis } & \multicolumn{2}{|l|}{ Model } \\
\hline & Significant features & p-value & AUROC (CI95\%) & p-value \\
\hline \multicolumn{5}{|c|}{ Sampling } \\
\hline \multirow[t]{2}{*}{ dt2 } & $\mathrm{K}^{\text {trans}} \_$GLRLM_Run entropy & 0.021 & $0.85(0.68-1.00)$ & 0.021 \\
\hline & AUC_GLRLM_Run entropy & 0.050 & & \\
\hline \multirow[t]{5}{*}{ dt4 } & AUC_GLRLM_Run entropy & 0.050 & $0.84(0.62-1.00)$ & 0.026 \\
\hline & $\mathrm{K}^{\text {trans}}$ FOS_Inter-quartile range & 0.026 & & \\
\hline & $\mathrm{K}^{\text {trans}}$ FOS_Standard deviation & 0.013 & & \\
\hline & $\mathrm{K}^{\text {trans}}$ GLRLM_Long run emphasis & 0.021 & & \\
\hline & 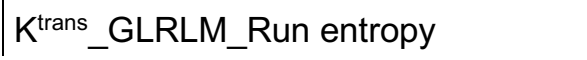 & 0.032 & & \\
\hline dt6 & AUC_GLRLM_Run entropy & 0.050 & $0.77(0.54-1.00)$ & 0.074 \\
\hline dt8 & AUC_GLCM_Correlation & 0.032 & $0.83(0.61-1.00)$ & 0.032 \\
\hline dt10 & $\mathrm{K}^{\text {trans}}{ }_{\text {GLRLM_Long run emphasis }}$ & 0.032 & $0.83(0.63-1.00)$ & 0.032 \\
\hline \multirow[t]{3}{*}{ dt12 } & AUC_GLCM_Correlation & 0.040 & $0.83(0.58-1.00)$ & 0.032 \\
\hline & AUC_GLRLM_Run entropy & 0.032 & & \\
\hline & 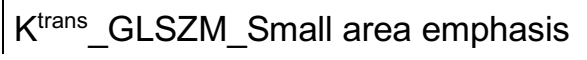 & 0.050 & & \\
\hline dt20 & AUC_GLRLM_Run entropy & 0.049 & $0.81(0.58-1.00)$ & 0.121 \\
\hline \multicolumn{5}{|c|}{ Truncating } \\
\hline \multirow[t]{2}{*}{ T3'00 } & AUC_GLRLM_Run entropy & 0.050 & $0.88(0.71-1.00)$ & 0.021 \\
\hline & 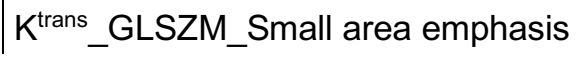 & 0.021 & & \\
\hline \multirow[t]{2}{*}{ T4'00 } & AUC_GLRLM_Run entropy & 0.050 & $0.90(0.74-1.00)$ & 0.018 \\
\hline & $K^{\text {trans}}$ GLSZM_Small area emphasis & 0.016 & & \\
\hline T5'00 & AUC_GLRLM_Run entropy & 0.050 & $0.77(0.54-1.00)$ & 0.074 \\
\hline
\end{tabular}

NOTE. Abbreviations: AUROC: area under the receiver operating characteristics curve; CI95\%: 95\% confidence interval; GL: grey level; GLCM: grey level co-occurrence matrix; GLRLM: grey level run length matrix; GLSZM: grey level size zone matrix; 'dtx' corresponds to a sampling of ' $x$ ' seconds; 'Ty' corresponds to a scan duration of ' $y$ ' minutes. 


\section{FIGURE LEGENDS}

FIGURE 1: Reconstruction of data acquisition. (a) Retrospective downsampling and truncating were performed to obtain post-processed data with a temporal resolution of $2 \mathrm{~s}(: \mathrm{dt} 2), 4 \mathrm{~s}(: \mathrm{dt} 4), 6 \mathrm{~s}(: \mathrm{dt} 6 \mathrm{~s}) \ldots$ and $20 \mathrm{~s}(: \mathrm{dt} 20)$, and with a scan duration of $4 \min \left(: 4^{\prime} 00\right.$ - with a temporal resolution of $\left.6 \mathrm{~s}\right)$ and $3 \mathrm{~min}$ (: T3'00 - with a temporal resolution of 6s). Of note, the baseline was not re-sampled. (b) The same voxels in the same artery were used to determine the artery input function (AIF) that was used in the extended Tofts model. However, AIFs were also truncated and downsampled leading to variations in time to reach the peak (from $36 \mathrm{~s}$ to $54 \mathrm{~s}$ ) and in maximum signal intensity of the peak (from 319 to 396.8) in this example of highgrade synovial sarcoma of the popliteal region. (c) Next, parametric maps of $\mathrm{K}^{\text {trans }}$ (: influx volume transfer constant) and (d) AUC (: area under the time intensity curves at $90 \mathrm{~s}$ after arrival of the contrast agent bolus in the acquisition volume) were generated as functions of ' $\mathrm{dt}$ ' and ' $\mathrm{T}$ '.

\section{FIGURE 2: Linear correlations between texture features from DCE-MRI} parametric maps and temporal resolution. Only significant correlations for AUC (a) and $\mathrm{K}^{\text {trans }}$ (b) after corrections to allow multiple comparisons are shown with their p-value. Sampling is given in seconds. Texture features have arbitrary units. Abbreviations: GL: grey level; GLSLZM: grey level size zone matrix.

FIGURE 3: Illustrated case of the influence of temporal parameters on DCEMRI parametric maps and texture features of sarcoma. A 63 years old male with a high-grade, deep and superficial, myxofibrosarcoma of the left thigh underwent his baseline MRI examination including conventional sequences (a) and DCE-MRI acquisition. (b) The whole tumor volume was manually segmented, slice-by-slice, on the last phase of the DCE-MRI acquisition, i.e. 300s after Gadolinium chelates intravenous injection. (c) The AUC (: area under the time intensity curve at $90 \mathrm{~s}$ after arrival of the contrast agent bolus in the acquisition volume, units: $\mathrm{mmol} / \mathrm{L} / \mathrm{s}$, on the right) and $\mathrm{K}^{\text {trans }}$ (influx volume transfer constant, units: /s, on the left) parametric maps were reconstructed with the different scan durations and temporal resolutions. After the whole tumor volume segmentation, frequency histograms were reconstructed for (d) AUC depending on the different temporal resolution, (e) Ktrans depending on the different temporal resolution, and (f) Ktrans according to the 
different scan duration. Abbreviation: STIR T2-WI: short time inversion recovery T2weighted imaging, T2-WI: T2 weighted imaging; FS CE-T1-WI: fat saturation contrast enhanced T1 weighted imaging; DCE-MRI $t=300 \mathrm{~s}$ : last phase of the dynamic contrast enhanced MRI, on which was segmented the tumor volume, 'dtx' corresponds to a sampling of ' $\mathrm{x}$ ' seconds; 'Ty' corresponds to a scan duration of ' $y$ ' minutes.

FIGURE 4 Coefficient of variation of the texture features extracted from DCEMRI depending on temporal parameters. Influence of the temporal resolution of DCE-MRI acquisition (: sampling) on the dispersion of each texture feature from (a) AUC (: area under the time intensity curve) and (b) $\mathrm{K}^{\text {trans }}$ (: influx volume transfer constant) maps. (c) Influence of the scan duration (: truncating) on the dispersion of each texture feature extracted from $\mathrm{K}^{\text {trans }}$ map. Results are given with standard deviation. GL: grey level.

\section{SUPPLEMENTARY DATA}

Supplementary Data 1. Definition of the texture features

Supplementary Data 2. Assessment of linear correlations of all the texture features with a significant influence of temporal parameters according to repeated measures ANOVA (or non-parametric equivalent).

Supplementary Data 3. Coefficient of variations with standard deviation of all the texture features.

Supplementary Data 4. Univariate analysis of the associations between texture features and responses to chemotherapy for each model (depending on temporal resolution and scan duration).

Supplementary Data 5. Comparisons of the AUROC of the different models, depending on scan duration and temporal resolution 
FIGURE 1

\section{a}

baseline

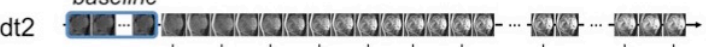

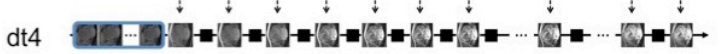

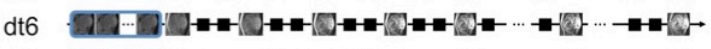

dt8

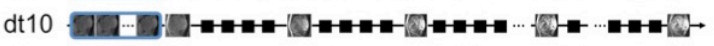

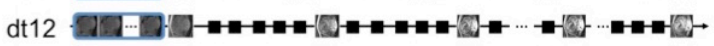

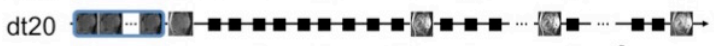

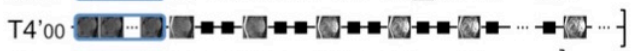

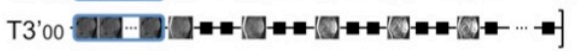

C

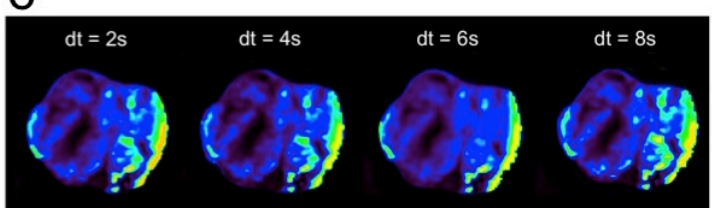

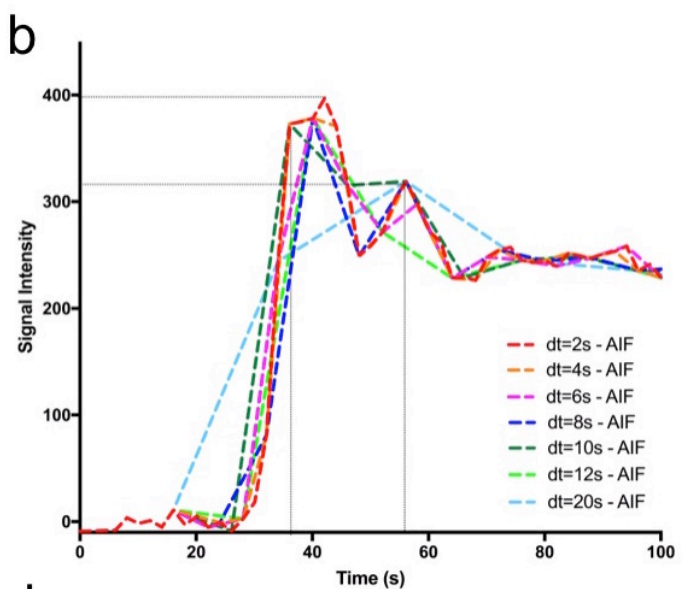

d

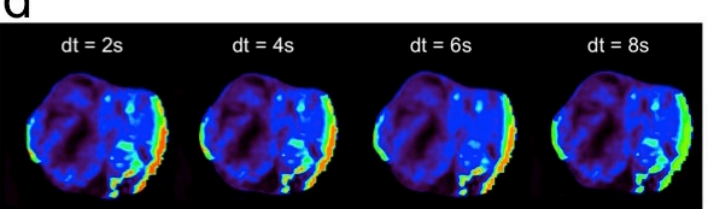

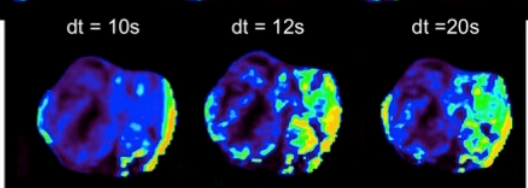
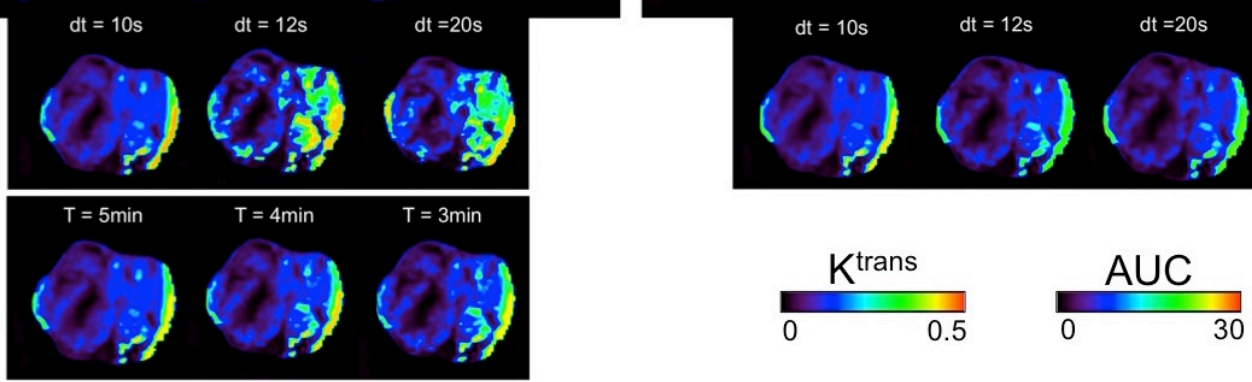
FIGURE 2.
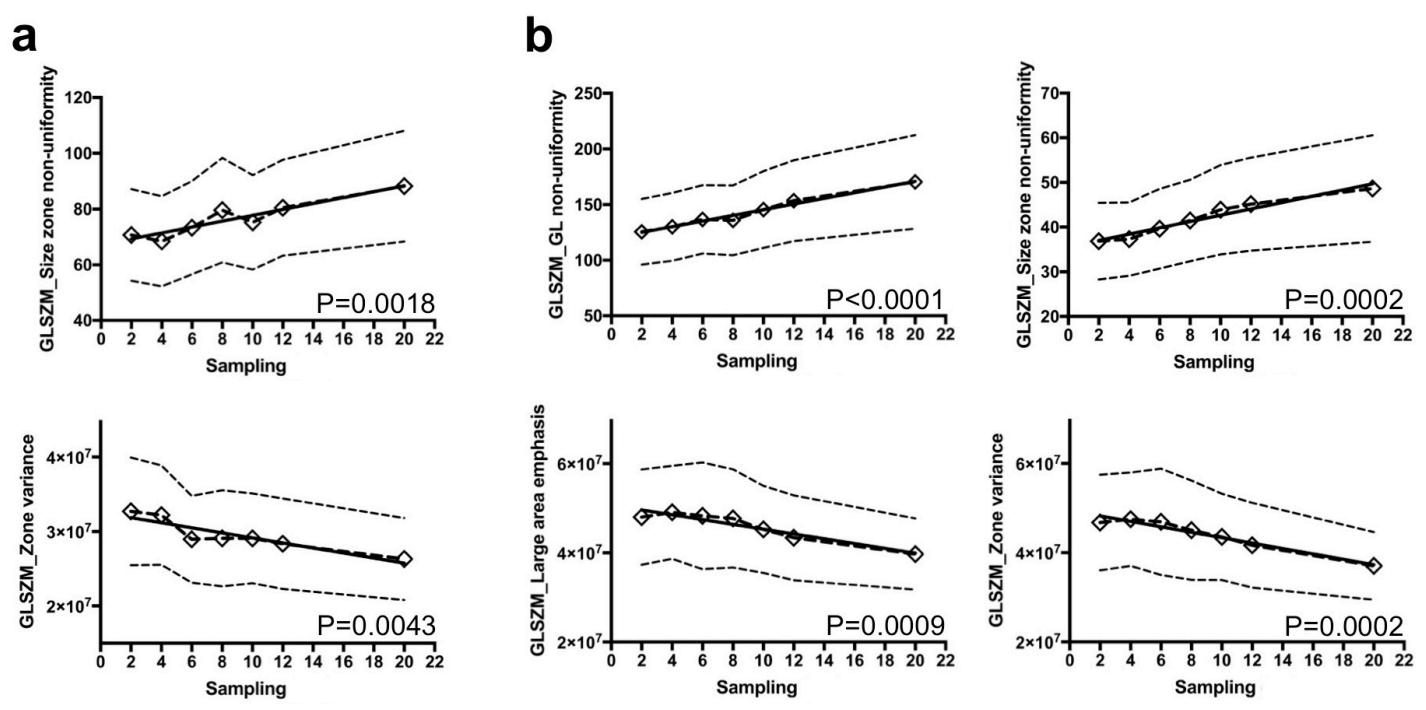
FIGURE 3.
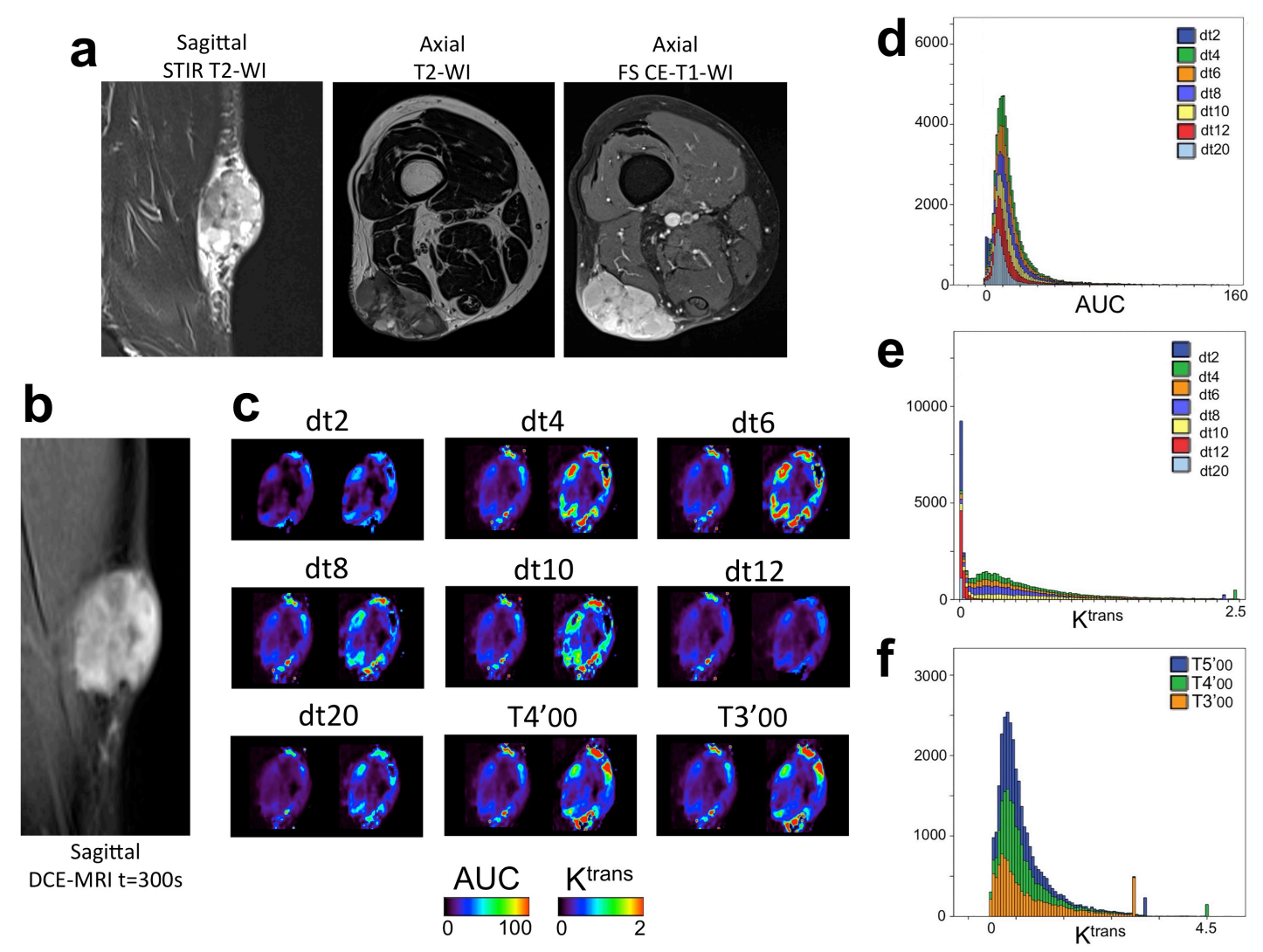
FIGURE 4.

a

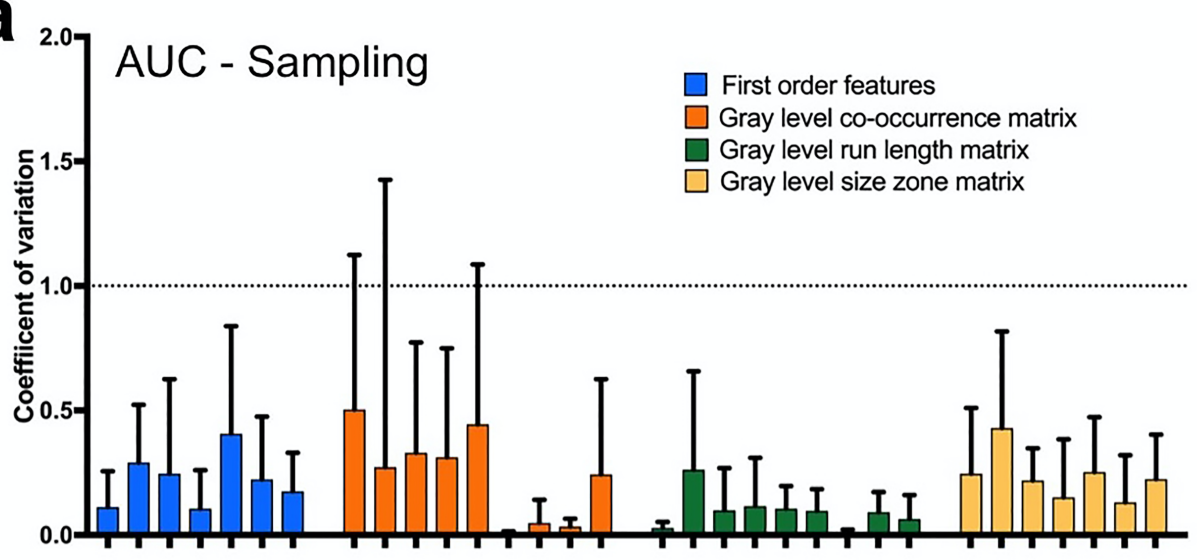

b

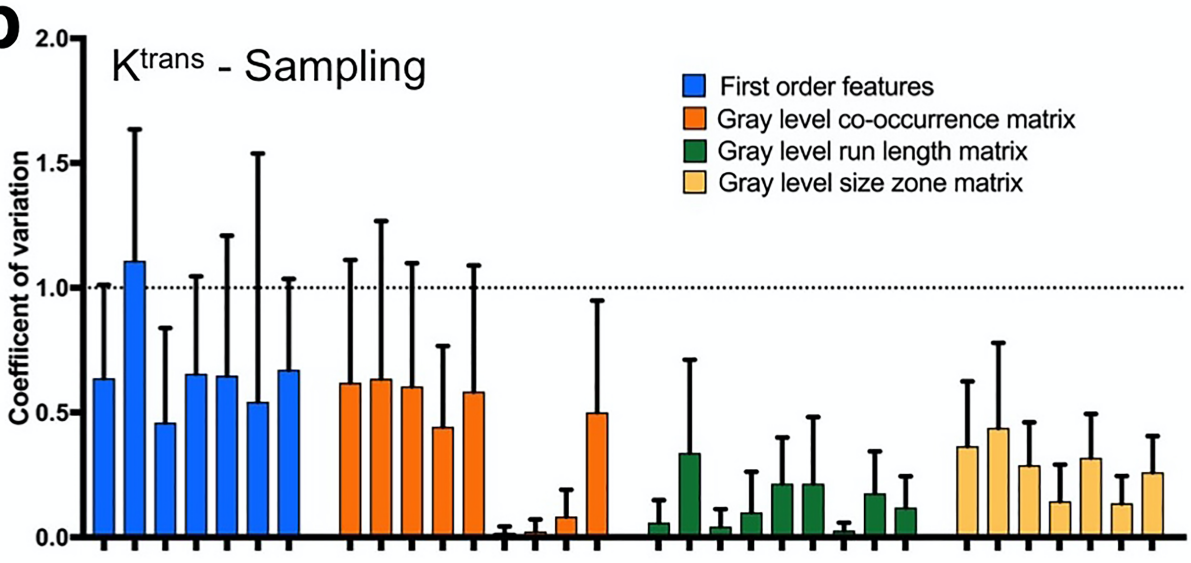

C

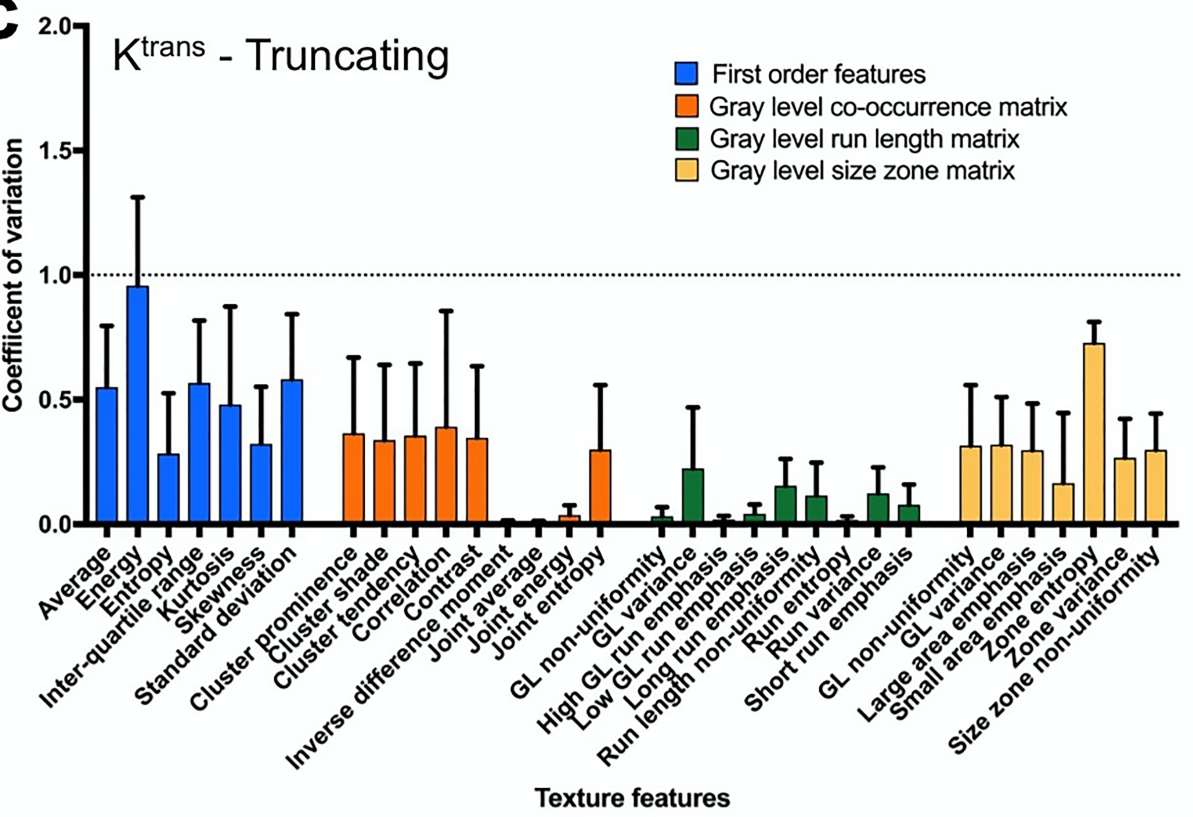

